J. Pachl

Systemtechnik

des Schienenverkehrs 


\section{Systemtechnik des Schienenverkehrs}

Von Professor Dr.-Ing. Jörn Pachl

Technische Universität Braunschweig

2., überarbeitete und erweiterte Auflage

Mit 155 Bildern und 13 Tabellen 
ISBN 978-3-519-16383-1 ISBN 978-3-322-96776-3 (eBook)

DOI 10.1007/978-3-322-96776-3

Die Deutsche Bibliothek - CIP-Einheitsaufnahme

Ein Titeldatensatz für diese Publikation ist bei

Der Deutschen Bibliothek erhältlich

Das Werk einschlieBlich aller seiner Teile ist urheberrechtlich geschützt. Jede Verwertung außerhalb der engen Grenzen des Urheberrechtsgesetzes ist ohne Zustimmung des Verlages unzulässig und strafbar. Das gilt besonders für Vervielfältigungen, Übersetzungen, Mikroverfilmungen und die Einspeicherung und Verarbeitung in elektronischen Systemen.

(C) 2000 B. G. Teubner Stuttgart • Leipzig

Gesamtherstellung: Präzis-Druck GmbH, Karlsruhe

Umschlaggestaltung: Peter Pfitz, Stuttgart 


\section{Vorwort}

Der Eisenbahnverkehr hat in den letzten Jahren sowohl national als auch international wieder erheblich an Bedeutung gewonnen. In allen Bereichen des Eisenbahnwesens besteht dadurch ein verstärkter Bedarf an solide ausgebildeten Fach- und Führungskräften. Während für einzelne Fachsparten der Bahntechnik bereits ein ansprechendes Angebot an Hochschullehrbüchern zur Verfügung steht, fehlt seit längerem eine fachübergreifende Beschreibung der grundsätzlichen systemtechnischen Zusammenhänge des Schienenverkehrs. Diese Lücke möchte das vorliegende Lehrbuch schließen. Das Buch wendet sich in erster Linie an Studenten technischer Studiengänge an Hoch- und Fachhochschulen, insbesondere Verkehrswesen, Bauingenieurwesen, Elektrotechnik, Automatisierungstechnik und Informatik, die sich im Rahmen ihres Haupt- oder Vertiefungsstudiums ein Grundwissen über die Systemtechnik der Eisenbahn aneignen möchten. Es wendet sich aber ebenfalls an Teilnehmer von Trainee- und Weiterbildungsprogrammen der Eisenbahnunternehmen und der Bahnindustrie und soll auch fachlichen Quereinsteigern ermöglichen, sich mit dem System Bahn vertraut zu machen.

Im Unterschied zu Lehrwerken, die sich einer anlagenorientierten Beschreibung einzelner Komponenten der Bahntechnik widmen, besteht das Anliegen des vorliegenden Werkes in einer prozessorientierten Betrachtung des Systems Bahn. Nach einer Einführung in die Begriffswelt des Schienenverkehrs folgt zunächst eine kurze Abhandlung der für die Systemtechnik relevanten fahrdynamischen Grundlagen. Den Hauptteil des Buches bilden die Abschnitte zur Regelung und Sicherung der Zugfolge und zur Steuerung und Sicherung der Fahrwegelemente. In diesen Abschnitten werden die maßgebenden Systemeigenschaften des Schienenverkehrs einer eingehenden Betrachtung unterzogen. Darauf unmittelbar aufbauend folgt ein Kapitel zur Leistungsuntersuchung von Eisenbahnbetriebsanlagen, in dem der Versuch unternommen wird, traditionelle Betrachtungsweisen mit neuesten Erkenntnissen der Eisenbahnbetriebswissenschaft zu verbinden. Den Abschluss bilden Kapitel zu Fragen der Fahrplankonstruktion und der Betriebssteuerung.

Im Eisenbahnwesen existiert eine umfangreiche historisch gewachsene Begriffswelt, die sich in dieser Form in anderen Sparten der Technik und des Ver- 
kehrs nicht findet. Dem mit dieser Begriffswelt nicht vertrauten fachlichen Neueinsteiger ist das Verständnis systemtechnischer Zusammenhänge mitunter erheblich erschwert. Aus diesem Grunde wurde in den Anhang des Buches ein Glossar mit Kurzdefinitionen von mehr als 200 Grundbegriffen des Eisenbahnwesens aufgenommen.

In Inhalt und Gestaltung dieses Werkes sind in maßgebender Weise die Erfahrungen aus der Lehrtätigkeit am Institut für Eisenbahnwesen und Verkehrssicherung der TU Braunschweig sowie aus Lehrveranstaltungen für die Aus- und Weiterbildung von Fachkräften der Bahnindustrie eingeflossen. In der vorliegenden 2. Auflage wurden einige Ergänzungen und Aktualisierungen vorgenommen, wobei insbesondere die Anregungen aus dem Leserkreis Berücksichtigung fanden. Vorschläge für weitere Verbesserungen und Hinweise auf Fehler sind jederzeit willkommen.

Mein Dank gebührt dem Verlag und seinen Mitarbeitern, insbesondere Herrn Dr. Schlembach und Frau Rodeit, für die konstruktive Zusammenarbeit, die sorgfältige Herstellung und die gute Ausstattung dieses Buches.

Braunschweig, Dezember 1999

Jörn Pachl

Für Anregungen und Hinweise ist der Autor im Internet unter folgenden Adressen zu erreichen: http://www.ivev.bau.tu-bs.de/ pachl/ email: joern.pachl@gmx.de 


\section{Inhalt}

1 Grundbegriffe des Schienenverkehrs.....................................................

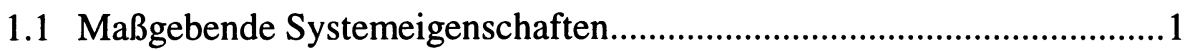

1.2 Gesetzliche Grundlagen für Bau und Betrieb von Bahnen .......................3

1.3 Grundlegende Begriffe und Definitionen..................................................6

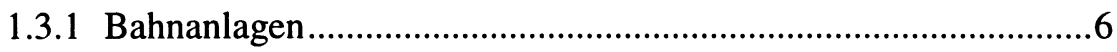

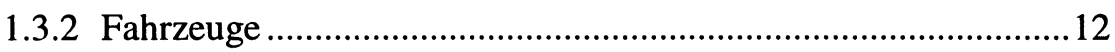

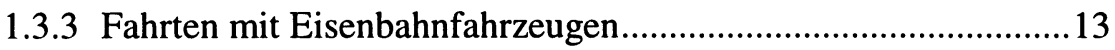

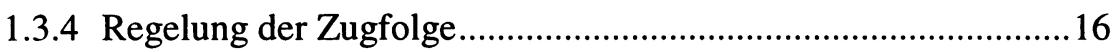

1.3.5 Abweichende Begriffswelten im Ausland ..................................18

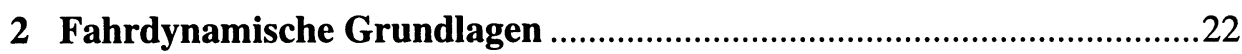

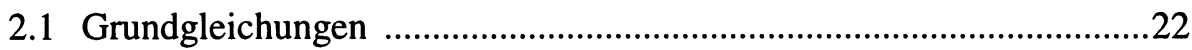

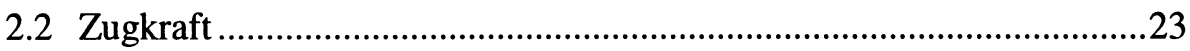

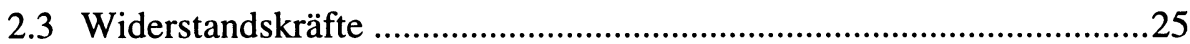

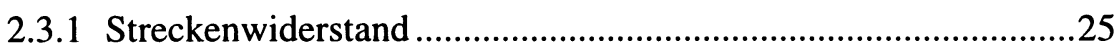

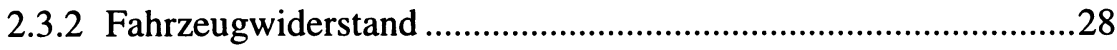

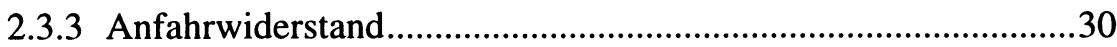

2.4 Steigungs-Geschwindigkeits-Diagramm ................................................

2.5 Fahrzeitermittlung ................................................................................33

3 Regelung und Sicherung der Zugfolge....................................................38

3.1 Theoretische Abstandshaltevorschriften ................................................38

3.1.1 Fahren im relativen Bremswegabstand .........................................38

3.1.2 Fahren im absoluten Bremswegabstand .......................................39 


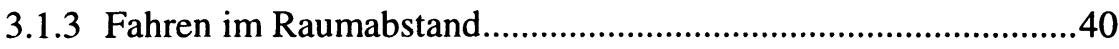

3.2 Abstandshaltetechniken im Schienenverkehr......................................41

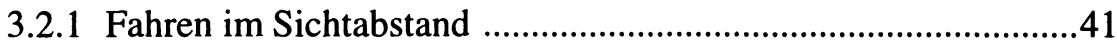

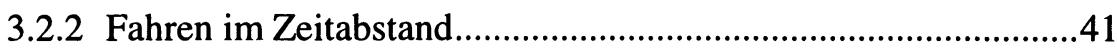

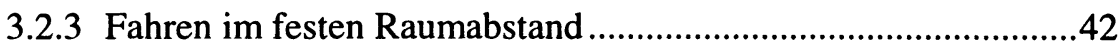

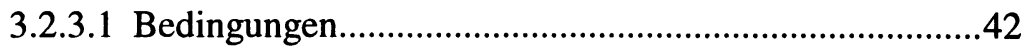

3.2.3.2 Ortsfeste Signalisierung ................................................43

3.2.3.3 Sperrzeitenbild .............................................................50

3.2.3.4 Leistungssteigerung durch Signalisierung verkürzter Blockabschnitte............................................53

3.3 Sicherung des Fahrens im festen Raumabstand ....................................57

3.3.1 Verfahren ohne technische Sicherung ……..............................57

3.3.1.1 Verfahren ohne technische Sicherung mit örtlicher Fahrdienstleitung .............................................58

3.3.1.2 Verfahren ohne technische Sicherung mit zentraler Fahrdienstleitung ..........................................60

3.3.2 Technische Sicherungsverfahren ............................................61

3.3.2.1 Begriff des Streckenblocks .............................................61

3.3.2.2 Gestaltung der Blocklogik.............................................61

3.3.2.3 Technische Realisierung des Streckenblocks ................65

3.3.2.4 Satellitengestützte Sicherung der Zugfolge als Alternative zum Streckenblock auf Nebenstrecken .......73

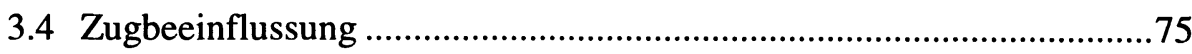

3.4.1 Arten von Zugbeeinflussungsanlagen ..........................................75

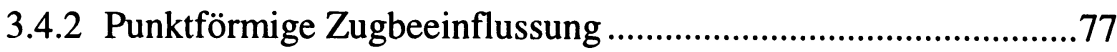

3.4.2.1 Zugbeeinflussung mit mechanischer und elektrischer (galvanischer) Informationsübertragung........................77

3.4.2.2 Induktive Zugbeeinflussung (Indusi) .............................77

3.4.2.3 Magnetische Zugbeeinflussung.......................................82

3.4.2.4 Zugbeeinflussung mit punktförmigen Datenübertragungssystemen auf Transponderbasis.........................82

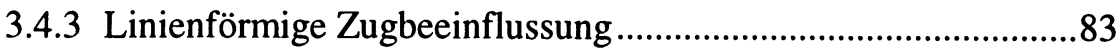

3.4.4 Vereinheitlichung der Zugbeeinflussung in Europa ...................87 
3.5 Funkbasierte Verfahren zur Zugfolgesicherung...................................90

4 Steuerung und Sicherung der Fahrwegelemente ..................................94

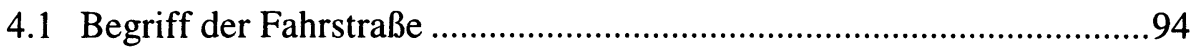

4.2 Kriterien für die Sicherung einer Fahrstraße........................................96

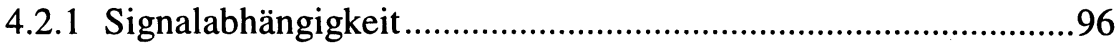

4.2.2 Fahrstraßenverschluss und Fahrstraßenfestlegung .....................98

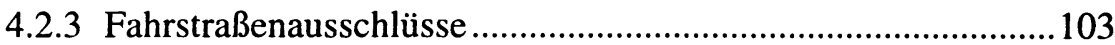

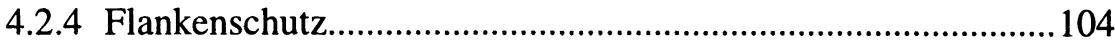

4.2.4.1 Flankenschutz gegen feindliche Zugfahrten ................105

4.2.4.2 Flankenschutz gegen feindliche Rangierfahrten und unbeabsichtigt ablaufende Wagen ................................105

4.2.4.3 Flankenschutz gegen das Strecken von Zügen..............106

4.2.4.4 Erfordernis von Schutzweichen ...................................107

4.2.4.5 Besonderheiten der Anordnung von Flankenschutz-

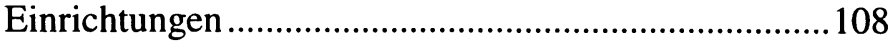

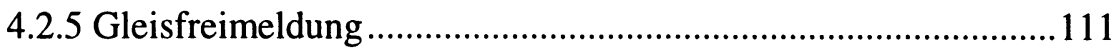

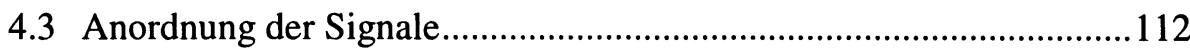

4.3.1 Verwendung der Hauptsignale ..............................................112

4.3.2 Bezeichnung der Hauptsignale ...................................................115

4.3.3 Abstand vom Gefahrpunkt ...................................................116

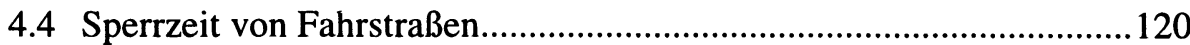

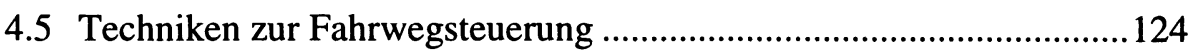

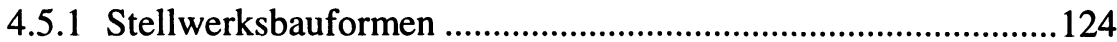

4.5.1.1 Mechanische Stellwerke ............................................124

4.5.1.2 Elektromechanische und elektropneumatische

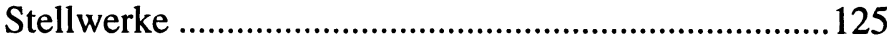

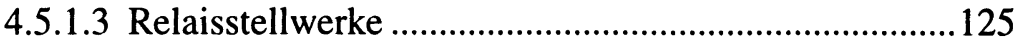

4.5.1.4 Elektronische Stellwerke (ESTW) ...............................126

4.5.2 Abbildung der Fahrstraßenlogik in Stellwerken ........................126

4.5.2.1 Tabellarische Fahrstraßenlogik (Verschlusstabelle)....126

4.5.2.2 Geografische Fahrstraßenlogik (Spurplanprinzip).......128 
4.5.3 Abhängigkeiten zwischen Bedienbereichen .............................131

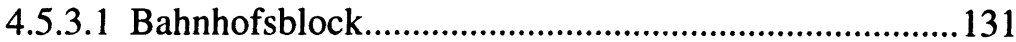

4.5.3.2 Abhängigkeiten zwischen Bedienbereichen in elektronischen Stellwerken ..........................................134

4.5.3.3 Nahbedienungsfreigaben..............................................135

4.5.4 Streckensicherung mit den Mitteln der Fahrstraßentechnik .....135

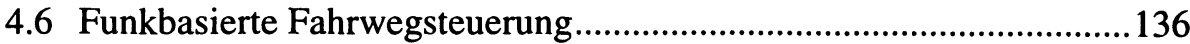

5 Leistungsuntersuchung von Eisenbahn-Betriebsanlagen ....................140

5.1 Leistungsverhalten und Leistungsfähigkeit.......................................140

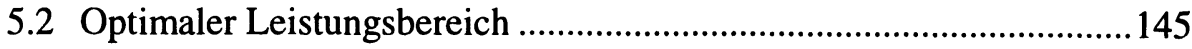

5.3 Methodik der Leistungsuntersuchungen .............................................149

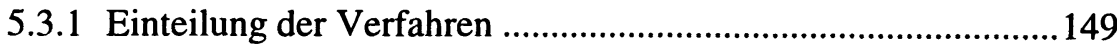

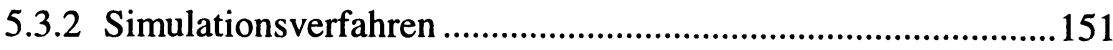

5.3.3 Analytische Untersuchung von Strecken ..................................154

5.3.4 Analytische Untersuchung von Knoten .....................................163

5.3.4.1 Analytische Untersuchung von Fahrstraßenknoten ......164

5.3.4.2 Vereinfachte Verfahren zur Abbildung der betrieblichen Möglichkeiten eines Fahrstraßenknotens..........166

5.3.4.3 Bemessung von Gleisgruppen .....................................176

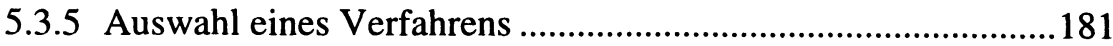

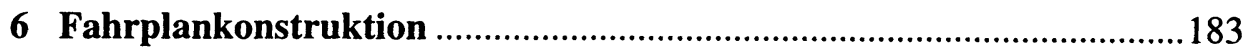

6.1 Darstellungsformen des Fahrplans für das Trassenmanagement .........184

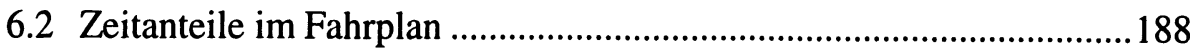

6.2.1 Bestandteile der Beförderungszeit eines Zuges ........................188

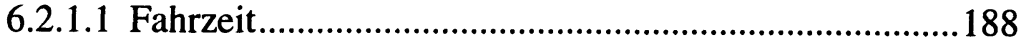

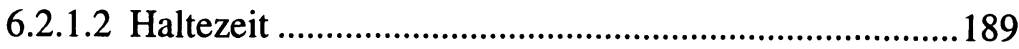

6.2.2 Zeitanteile zwischen den Zugfahrten .....................................191

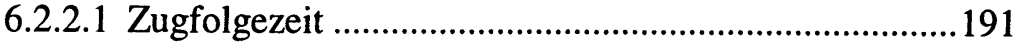

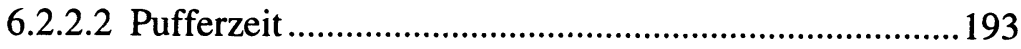

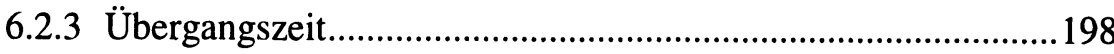




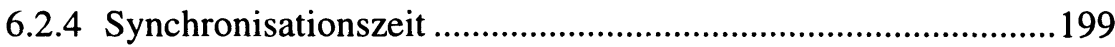

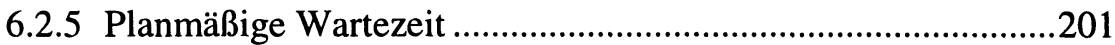

6.3 Verfahren zur Fahrplankonstruktion .................................................203

6.3.1 Manuelle Fahrplankonstruktion ..............................................204

6.3.2 Rechnergestützte Fahrplankonstruktion..................................206

6.4 Fahrplanqualität und Fahrplanleistung ...............................................208

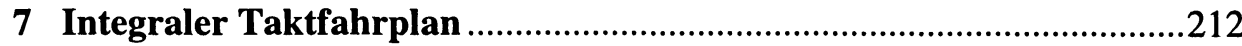

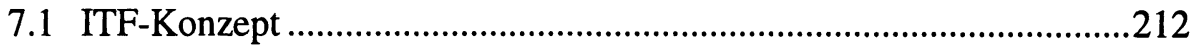

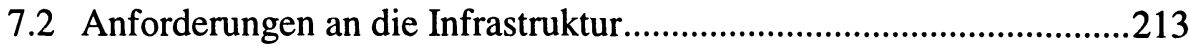

7.2.1 Strecken-Infrastruktur für feste Taktlagen ...............................216

7.2.2 Strecken-Infrastruktur für wechselnde Taktlagen.....................221

7.2.3 Infrastruktur der Knoten ...........................................................221

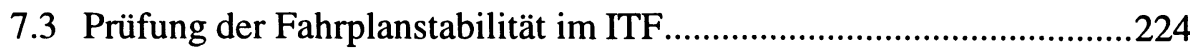

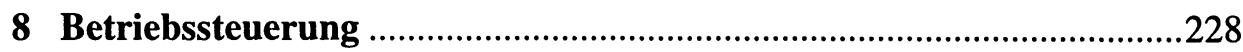

8.1 Organisation der Fahrdienstleitung ...................................................228

8.2 Arbeitshilfen bei manueller Betriebssteuerung ..................................229

8.3 Betriebsleittechnik zur Unterstützung der Betriebsssteuerung ...........230

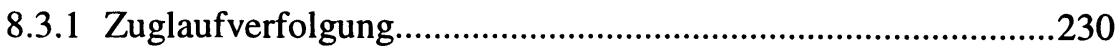

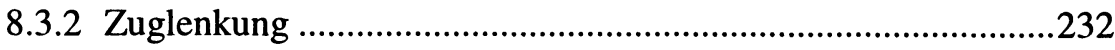

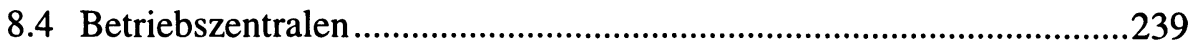

8.4.1 Aufteilung der betrieblichen Funktionalität in einer

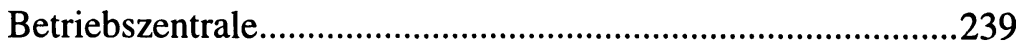

8.4.2 Rückfallebenen für den Störungsfall ........................................243

8.4.3 Grundlagen der rechnergestützten Disposition ..........................246 


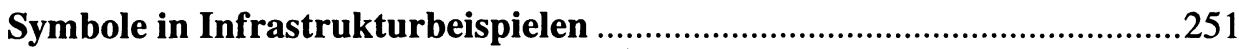

Glossar

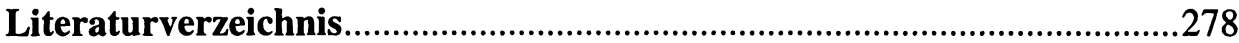

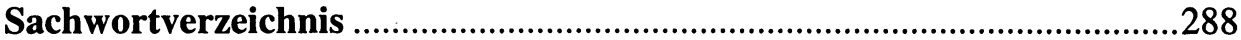

